\title{
Giant enhancement of the controllable in-plane anisotropy of biased isotropic noncentrosymmetric materials with epsilon-negative multilayers
}

C. A. Valagiannopoulos, N. L. Tsitsas, and A. Lakhtakia

Citation: J. Appl. Phys. 121, 063102 (2017); doi: 10.1063/1.4975482

View online: http://dx.doi.org/10.1063/1.4975482

View Table of Contents: http://aip.scitation.org/toc/jap/121/6

Published by the American Institute of Physics

\section{Articles you may be interested in}

Effects of photon reabsorption phenomena in confocal micro-photoluminescence measurements in crystalline silicon

J. Appl. Phys. 121, 063101063101 (2017); 10.1063/1.4975476

Ultra-thin narrow-band, complementary narrow-band, and dual-band metamaterial absorbers for applications in the $\mathrm{THz}$ regime

J. Appl. Phys. 121, 063103063103 (2017); 10.1063/1.4975687

Plasma potential of a moving ionization zone in DC magnetron sputtering

J. Appl. Phys. 121, 063302063302 (2017); 10.1063/1.4974944

Long-range Tamm surface plasmons supported by graphene-dielectric metamaterials

J. Appl. Phys. 121, 033101033101 (2017); 10.1063/1.4973900

\section{A.P $\mid \begin{aligned} & \text { Journal of } \\ & \text { Applied Physics }\end{aligned}$}

INTRODUCING INVITED PERSPECTIVES

Ultrafast magnetism and $\mathrm{THz}$ spintronics

Authors: Jakob Walowski and Markus Münzenberg 


\title{
Giant enhancement of the controllable in-plane anisotropy of biased isotropic noncentrosymmetric materials with epsilon-negative multilayers
}

\author{
C. A. Valagiannopoulos, ${ }^{1}$ N. L. Tsitsas, ${ }^{2}$ and A. Lakhtakia ${ }^{3}$ \\ ${ }^{1}$ Department of Physics, School of Science and Technology, Nazarbayev University, 53 Qabanbay Batyr Ave, \\ Astana KZ-010000, Kazakhstan \\ ${ }^{2}$ Department of Informatics, Aristotle University of Thessaloniki, Thessaloniki GR-54124, Greece \\ ${ }^{3}$ Department of Engineering Science and Mechanics, Pennsylvania State University, University Park, \\ Pennsylvania 16802-6812, USA
}

(Received 20 October 2016; accepted 23 January 2017; published online 9 February 2017)

\begin{abstract}
Giant in-plane anisotropy can be exhibited by a finitely thick periodic multilayer comprising bilayers of an isotropic noncentrosymmetric material and a non-dissipative isotropic medium of negative permittivity, when a dc electric field is applied in the thickness direction. Compared to a homogeneous layer of the noncentrosymmetric material with the same thickness as the periodic multilayer, the latter exhibits an effective in-plane anisotropy that can be three orders larger in magnitude. This enhancement gets more substantial at higher frequencies and is electrically controllable. The incorporation of dissipation reduces the enhancement of the effective in-plane anisotropy, which nevertheless remains significant. We expect the finitely thick periodic multilayer to be useful as a polarization transformer or a modulator in the terahertz regime fully controllable via external dc bias. Published by AIP Publishing. [http://dx.doi.org/10.1063/1.4975482]
\end{abstract}

\section{INTRODUCTION}

Isotropic but noncentrosymmetric materials such as cadmium telluride (CdTe), cadmium sulfide (CdS), gallium arsenide (GaAs), and zinc telluride (ZnTe) possess a zincblende (cubic) crystalline structure. ${ }^{1}$ Therefore, in the midwavelength infrared (MWIR) and long-wavelength infrared regimes, these materials display an electro-optic effect discovered by Friedrich Pockels (1906). Of the four materials named previously, CdTe has the highest electro-optic coefficient. $^{2-4}$ The Pockels effect, as it is usually called, allows electrical control of optical response characteristics, especially for transmission ${ }^{5}$ but also for reflection. ${ }^{6}$ Bulk CdTe crystals with high resistivity $\left(>10^{9} \Omega \mathrm{cm}\right)$ are successfully used in intracavity electro-optic modulators for Q-switched $\mathrm{CO}_{2}$ lasers at $10.2 \mu \mathrm{m}$ wavelength. ${ }^{7}$

When an isotropic noncentrosymmetric material is subjected to a dc electric field $\mathbf{E}_{\mathrm{dc}}$, the medium acts like an orthorhombic dielectric material. ${ }^{2-4}$ In the Cartesian coordinate system $\left(x_{1}, x_{2}, x_{3}\right)$ with the coordinate axes aligned with the crystallographic axes and $\left\{\hat{\mathbf{x}}_{1}, \hat{\mathbf{x}}_{2}, \hat{\mathbf{x}}_{3}\right\}$ is the right-handed triad of mutually orthogonal unit vectors, the relative permittivity dyadic $\underline{\varepsilon}_{\mathrm{pc}}$ of the material may be stated as ${ }^{6}$

$$
\underline{\varepsilon}_{\mathrm{pc}}=\chi\left(\hat{\mathbf{x}}_{1} \hat{\mathbf{x}}_{1}+\hat{\mathbf{x}}_{2} \hat{\mathbf{x}}_{2}\right)+\alpha\left(\hat{\mathbf{x}}_{1} \hat{\mathbf{x}}_{2}+\hat{\mathbf{x}}_{2} \hat{\mathbf{x}}_{1}\right)+\psi \hat{\mathbf{x}}_{3} \hat{\mathbf{x}}_{3}
$$

when $\mathbf{E}_{\mathrm{dc}}=E_{\mathrm{dc}} \hat{\mathbf{x}}_{3}$. Here,

$$
\left.\begin{array}{l}
\chi=n_{\mathrm{pc}}^{2} /\left(1-r_{\mathrm{pc}}^{2} E_{\mathrm{dc}}^{2} n_{\mathrm{pc}}^{4}\right) \\
\alpha=-r_{\mathrm{pc}} E_{\mathrm{dc}} n_{\mathrm{pc}}^{4} /\left(1-r_{\mathrm{pc}}^{2} E_{\mathrm{dc}}^{2} n_{\mathrm{pc}}^{4}\right) \\
\psi=n_{\mathrm{pc}}^{2}
\end{array}\right\},
$$

where $n_{\mathrm{pc}}$ is the refractive index in the absence of dc electric field, $r_{\mathrm{pc}}$ is the sole electro-optic coefficient, and it has been assumed that $n_{\mathrm{pc}}^{2}\left|r_{\mathrm{pc}} E_{\mathrm{dc}}\right| \ll 1$; the assumption is justified in the next paragraph by using specific numerical values. Thus, there is not only transverse anisotropy with respect to the direction of $\mathbf{E}_{\mathrm{dc}}$, quantitated by the difference between $\chi$ and $\psi$, but there is also an in-plane anisotropy quantitated via $\alpha$ in the $x_{1} x_{2}$ plane. This in-plane anisotropy is commonly exploited in electro-optic modulators. ${ }^{5}$

However, $\alpha$ is at most of order $10^{-3}$ because the magnitude of $E_{\mathrm{dc}}$ cannot be very large (say, $\lesssim 10^{6} \mathrm{~V} \mathrm{~m}^{-1}$ ), $r_{\mathrm{pc}}$ is also a small number (say, $\lesssim 10^{-11} \mathrm{~m} \mathrm{~V}^{-1}$ ), and $n_{\mathrm{pc}} \lesssim 4$. This small magnitude of $\alpha$ necessitates the use of thick layers of isotropic noncentrosymmetric materials for significant phase differences between two polarization states to develop in THz devices and components. ${ }^{5,8}$

We hypothesized that one could alternatively use a multilayer comprising layers of an isotropic noncentrosymmetric material and an isotropic epsilon-negative (ENG) material $^{9-11}$ in order to enhance the in-plane anisotropy of the equivalent permittivity dyadic of the multilayer. Then, we would expect from homogenization formalisms ${ }^{12-14}$ applicable for electrically thin layers that the isotropic ENG material would diminish the effects of the parameters $\chi$ and $\psi$ that appear in the diagonal of $\underline{\varepsilon}_{p c}$ but not the effects of the anisotropy parameter $\alpha$ on the $x_{1} x_{2}$ plane. Of course, to achieve such an equivalence, the multilayer would have to possess a periodic structure where the isotropic noncentrosymmetric layers would alternate with the ENG ones.

Periodic multilayers have garnered renewed interest in recent years ${ }^{15-19}$ for obtaining unusual response characteristics. Therefore, we decided to examine the reflection and transmission of normally incident plane waves from a finitely thick periodic multilayer whose unit cell comprises an isotropic noncentrosymmetric slab and an isotropic ENG layer. ${ }^{20-24}$ Parenthetically, although ENG materials are dissipative, ${ }^{20}$ overcoming dissipation by using active-material components appears promising. ${ }^{11,25}$ Our main purpose was 
to examine our hypothesis that the finitely thick periodic multilayer can exhibit, in effect, a larger in-plane anisotropy compared to a single isotropic noncentrosymmetric layer of the same thickness as the periodic multilayer.

The plan of this paper is as follows. Section II describes the formulation and the solution of the boundary-value problem of reflection and transmission of a normally incident plane wave by a finite number of bilayers with properties described in the previous paragraph. We use a $2 \times 2$-matrix approach without any approximation ${ }^{26-28}$ or homogenization. ${ }^{12-14}$ Section III presents numerical results showing that enhancement of anisotropy can indeed be achieved by appropriately selecting the parameters of the finitely thick periodic multilayer. Furthermore, we consider certain actual plasmonic media exhibiting both frequency dispersion and dissipation and test their performance in enhancing the effective anisotropy of the structure. An $\exp (i 2 \pi f t)$ time-dependence is assumed and suppressed throughout the subsequent analysis, with $f$ as the linear frequency, $t$ as time, and $i=\sqrt{-1}$. The permittivity and permeability of free space are denoted by $\varepsilon_{0}$ and $\mu_{0}$, respectively. Vectors are in boldface and unit vectors are identified by a caret, while dyadics [Ref. 29, Chap. 1] are double underlined.

\section{BOUNDARY-VALUE PROBLEM}

A schematic of the boundary-value problem under consideration is depicted in Fig. 1. The half spaces $z<0$ and $z>N\left(w_{p}+w_{d}\right)$ are vacuous. The region $0<z<N\left(w_{p}\right.$ $\left.+w_{d}\right)$ is occupied by the finitely thick periodic multilayer composed of $N \geq 1$ unit cells. Each unit cell is a bilayer comprising (i) an isotropic noncentrosymmetric layer of thickness $w_{p}$ and relative permittivity dyadic $\underline{\varepsilon}_{\mathrm{pc}}$ and (ii) an isotropic ENG layer of thickness $w_{d}$ and relative permittivity dyadic $\varepsilon_{d} \underline{\underline{I}}$, where $\underline{\underline{I}}$ is the identity dyadic. Both component materials are assumed to have the same permeability as free space.

Instead of the Cartesian coordinate system $\left(x_{1}, x_{2}, x_{3}\right)$, let us use a Cartesian coordinate $\operatorname{system}(x, y, z)$ oriented such that $x=\left(x_{1}+x_{2}\right) / \sqrt{2}, y=\left(-x_{1}+x_{2}\right) / \sqrt{2}$, and $z=x_{3}$. Then, $\mathbf{E}_{\mathrm{dc}}=E_{\mathrm{dc}} \hat{\mathbf{z}}$ and Eq. (1) is rewritten as

$$
\underline{\varepsilon}_{\mathrm{pc}}=(\chi+\alpha) \hat{\mathbf{x}} \hat{\mathbf{x}}+(\chi-\alpha) \hat{\mathbf{y}} \hat{\mathbf{y}}+\psi \hat{\mathbf{z}} \hat{\mathbf{z}}
$$

Thus, despite the fact that the relative permittivity dyadic $\stackrel{\varepsilon}{f} \mathrm{pc}_{\mathrm{c}}$ looks complicated as stated in Eq. (1), the isotropic noncentrosymmetric material is simply an orthorhombic material when subjected to a unidirectional dc electric field.

Suppose that linearly polarized light is normally incident on the finitely thick periodic multilayer from the half space $z<0$. The electric field phasor of the incident light is oriented at an angle $\xi$ with respect to the $x$ axis in the $x y$ plane, as indicated in Fig. 1. The incident electric and magnetic field phasors are expressed as

$$
\left.\begin{array}{l}
\mathbf{E}^{\text {inc }}(z)=(\hat{\mathbf{x}} \cos \xi+\hat{\mathbf{y}} \sin \xi) \exp \left(-i k_{0} z\right) \\
\mathbf{H}^{\text {inc }}(z)=\eta_{0}^{-1}(-\hat{\mathbf{x}} \sin \xi+\hat{\mathbf{y}} \cos \xi) \exp \left(-i k_{0} z\right)
\end{array}\right\}, \quad z<0
$$

where $k_{0}=2 \pi / \lambda_{0}=2 \pi f \sqrt{\varepsilon_{0} \mu_{0}}$ denotes the free-space wavenumber with $\lambda_{0}$ being the free-space wavelength, and $\eta_{0}$ $=\sqrt{\mu_{0} / \varepsilon_{0}}$ is the intrinsic impedance of free space.

The reflected electric and magnetic field phasors in the same half space are expressed as

$\left.\begin{array}{l}\mathbf{E}^{\mathrm{ref}}(z)=\left[\hat{\mathbf{x}} R_{(+)} \cos \xi+\hat{\mathbf{y}} R_{(-)} \sin \xi\right] \exp \left(i k_{0} z\right) \\ \mathbf{H}^{\mathrm{ref}}(z)=\eta_{0}^{-1}\left[\hat{\mathbf{x}} R_{(-)} \sin \xi-\hat{\mathbf{y}} R_{(+)} \cos \xi\right] \exp \left(i k_{0} z\right)\end{array}\right\}, \quad z<0$,

respectively, where the reflection coefficients $R_{(+)}$and $R_{(-)}$ have to be determined. Likewise, the electric and magnetic field phasors transmitted in the half space $z>N\left(w_{p}+w_{d}\right)$ are expressed as

$$
\left.\begin{array}{l}
\mathbf{E}^{\text {trans }}(z)=\left[\hat{\mathbf{x}} T_{(+)} \cos \xi+\hat{\mathbf{y}} T_{(-)} \sin \xi\right] \exp \left[-i k_{0}(z-h)\right] \\
\mathbf{H}^{\text {trans }}(z)=\eta_{0}^{-1}\left[-\hat{\mathbf{x}} T_{(-)} \sin \xi+\hat{\mathbf{y}} T_{(+)} \cos \xi\right] \exp \left[-i k_{0}(z-h)\right]
\end{array}\right\},
$$

with unknown transmission coefficients $T_{(+)}$and $T_{(-)}$, while $h=N\left(w_{p}+w_{d}\right)$ is the overall thickness of the periodic multilayer.

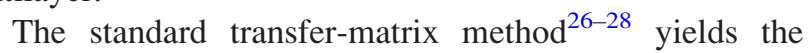
equation

$$
T_{( \pm)}\left[\begin{array}{c}
1 \\
\eta_{0}^{-1}
\end{array}\right]=\mathbf{M}_{( \pm)} \bullet\left[\begin{array}{c}
1+R_{( \pm)} \\
\eta_{0}^{-1}\left[1-R_{( \pm)}\right]
\end{array}\right]
$$

where the $2 \times 2$ matrices
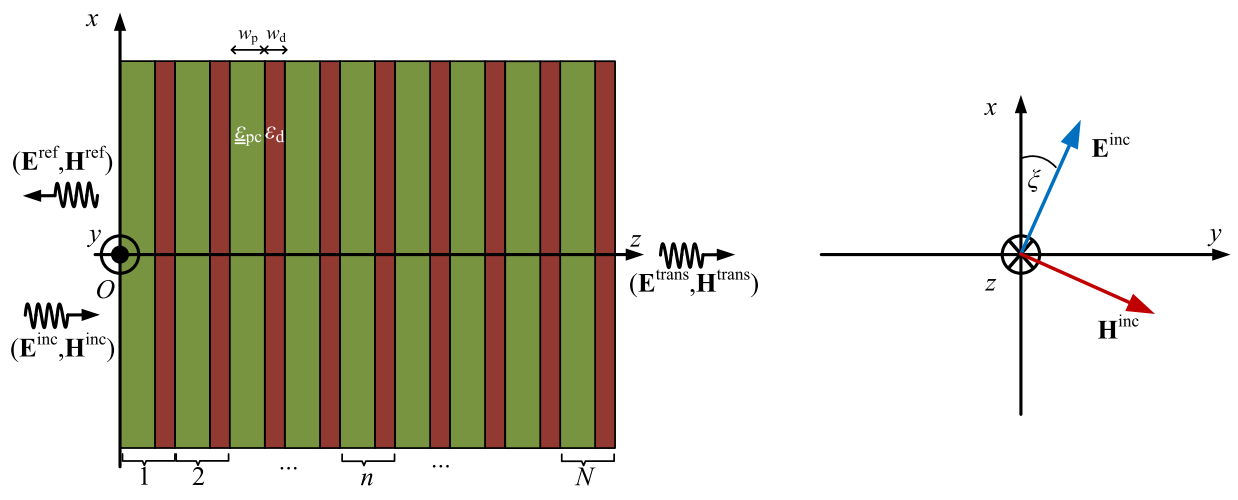

FIG. 1. Schematic of the boundaryvalue problem solved: A linearly polarized plane wave with polarization angle $\xi$ (defined in the inset at the right side) impinges normally on a finitely thick periodic multilayer composed of $N$ unit cells each one comprising an isotropic noncentrosymmetric layer and an isotropic ENG layer. The periodic multilayer is surrounded by vacuum on both sides. 


$$
\mathbf{M}_{( \pm)}=\left[\mathbf{P} \cdot \mathbf{Q} \cdot \mathbf{P}^{-1} \cdot \mathbf{U}_{( \pm)} \cdot \mathbf{V}_{( \pm)} \cdot \mathbf{U}_{( \pm)}^{-1}\right]^{N}
$$

are composed of the following $2 \times 2$ matrices:

$$
\begin{aligned}
& \mathbf{P}=\left[\begin{array}{cc}
1 & 1 \\
\eta_{0}^{-1} \sqrt{\varepsilon_{d}} & -\eta_{0}^{-1} \sqrt{\varepsilon_{d}}
\end{array}\right], \\
& \mathbf{Q}=\left[\begin{array}{cc}
\exp \left(-i k_{0} w_{d} \sqrt{\varepsilon_{d}}\right) & 0 \\
0 & \exp \left(i k_{0} w_{d} \sqrt{\varepsilon_{d}}\right)
\end{array}\right], \\
& \mathbf{U}_{( \pm)}=\left[\begin{array}{cc}
1 & 1 \\
\eta_{0}^{-1} \sqrt{\chi \pm \alpha} & -\eta_{0}^{-1} \sqrt{\chi^{ \pm} \alpha}
\end{array}\right], \\
& \mathbf{V}_{( \pm)}=\left[\begin{array}{cc}
\exp \left(-i k_{0} w_{p} \sqrt{\chi^{ \pm} \alpha}\right) & 0 \\
0 & \exp \left(i k_{0} w_{p} \sqrt{\chi^{ \pm \alpha}}\right)
\end{array}\right]
\end{aligned}
$$

\section{NUMERICAL RESULTS AND DISCUSSION}

We chose CdTe $\left(n_{\mathrm{pc}}=2.6\right.$ and $r_{\mathrm{pc}}=6.8 \times 10^{-12} \mathrm{~m}$ $\mathrm{V}^{-1}$ ) as the isotropic noncentrosymmetric material ${ }^{3}$ for all calculations reported here. The external dc electric field $E_{\mathrm{dc}}$ was confined to the interval $0 \leq E_{\mathrm{dc}} \leq 1 \mathrm{MV} \mathrm{m}^{-1}$ (maximum value much less than the CdTe breakdown limit $E_{\mathrm{dc}}=10 \mathrm{MV} \mathrm{m}^{-1}$ [Refs. 6 and 30]). For the chosen $E_{\mathrm{dc}^{-}}$ range, the off-diagonal element $\alpha$ is significantly smaller than $\chi$; hence, unless stated otherwise, numerical results are provided here for $E_{\mathrm{dc}}=1 \mathrm{MV} \mathrm{m}^{-1}$ when $\alpha$ has the largest value in the chosen $E_{\mathrm{dc}}$-range. The considered frequency range was $30 \mathrm{THz} \leq f \leq 90 \mathrm{THz}$ in which CdTe exhibits the Pockels effect. The thickness $w_{p}$ of the CdTe layers was confined to the interval $[20,300] \mathrm{nm}$; the lower bound was selected for the layers to be considered as material continua $^{31}$ and the upper bound to ensure that the CdTe layers would be electrically thin. ${ }^{12-14}$ The thickness $w_{d}$ of every ENG layer was chosen to lie within the interval $[100,200] \mathrm{nm}$, after noting that layers of such thicknesses can be made using molecular beam epitaxy, ${ }^{32}$ atomic layer deposition, ${ }^{33}$ and Langmuir-Blodgett methods. ${ }^{34}$

It should be remarked that, for the results presented in Sec. III B, we assumed the ENG material to be a nondissipative plasmonic material (i.e., $\operatorname{Re}\left[\varepsilon_{d}\right]<0$ and $\left.\operatorname{Im}\left[\varepsilon_{d}\right]=0\right)$ in order to examine the effective in-plane anisotropy of the finitely thick periodic multilayer without being affected by attenuation through the ENG layers. ${ }^{35}$ An additional reason that ENG materials with low dissipation or/and increased breakdown strength are needed is the immunity of their electromagnetic properties to the external dc electric field $E_{\mathrm{dc}}$. For the results presented in Sec. III C, we incorporated both dissipation and dispersion in the ENG material. Let us finally note that the number $N$ of bilayers must not be too large in order to avoid numerical instabilities in the transfer-matrix method. ${ }^{36-38}$

\section{A. Metrics for effective in-plane anisotropy}

In order to examine the effective in-plane anisotropy of the finitely thick periodic multilayer, we partitioned the reflected and the transmitted electric field phasors as

$$
\left.\begin{array}{l}
\mathbf{E}^{\mathrm{ref}}(z)=\mathbf{E}_{\mathrm{co}}^{\mathrm{ref}}(z)+\mathbf{E}_{\text {cross }}^{\mathrm{ref}}(z) \\
\mathbf{E}^{\text {trans }}(z)=\mathbf{E}_{\mathrm{co}}^{\text {trans }}(z)+\mathbf{E}_{\text {cross }}^{\text {trans }}(z)
\end{array}\right\}
$$

where the components

$$
\left.\begin{array}{l}
\mathbf{E}_{\mathrm{co}}^{\mathrm{ref}}(z)=\left[R_{(+)} \cos ^{2} \xi+R_{(-)} \sin ^{2} \xi\right](\hat{\mathbf{x}} \cos \xi+\hat{\mathbf{y}} \sin \xi) \exp \left(i k_{0} z\right) \\
\mathbf{E}_{\mathrm{co}}^{\text {trans }}(z)=\left[T_{(+)} \cos ^{2} \xi+T_{(-)} \sin ^{2} \xi\right](\hat{\mathbf{x}} \cos \xi+\hat{\mathbf{y}} \sin \xi) \exp \left[-i k_{0}(z-h)\right]
\end{array}\right\}
$$

are polarized parallel to $\mathbf{E}^{\mathrm{inc}}(z)$, and the components

$$
\left.\begin{array}{l}
\mathbf{E}_{\text {cross }}^{\text {ref }}(z)=\left[R_{(-)}-R_{(+)}\right] \sin \xi \cos \xi(-\hat{\mathbf{x}} \sin \xi+\hat{\mathbf{y}} \cos \xi) \exp \left(i k_{0} z\right) \\
\mathbf{E}_{\text {cross }}^{\text {trans }}(z)=\left[T_{(-)}-T_{(+)}\right] \sin \xi \cos \xi(-\hat{\mathbf{x}} \sin \xi+\hat{\mathbf{y}} \cos \xi) \exp \left[-i k_{0}(z-h)\right]
\end{array}\right\}
$$

are cross-polarized with respect to $\mathbf{E}^{\text {inc }}(z)$. For crosspolarization being engendered by in-plane anisotropy, we formulated the total cross-polarized power density

$$
P_{\text {cross }}=\frac{1}{2 \eta_{0}}\left[\left|R_{(-)}-R_{(+)}\right|^{2}+\left|T_{(-)}-T_{(+)}\right|^{2}\right] \sin ^{2} \xi \cos ^{2} \xi .
$$

Noting that the incident power density $P_{\text {inc }}=1 / 2 \eta_{0}$, we devised the quantity

$m_{\mathrm{abs}}=\frac{P_{\text {cross }}}{P_{\text {inc }}}=\left[\left|R_{(-)}-R_{(+)}\right|^{2}+\left|T_{(-)}-T_{(+)}\right|^{2}\right] \sin ^{2} \xi \cos ^{2} \xi$ as an absolute metric of the effective in-plane anisotropy of the finitely thick periodic multilayer.

Let $R_{( \pm)}^{\prime}$ and $T_{( \pm)}^{\prime}$ be the values of the reflection and transmission coefficients, respectively, when every ENG layer in the finitely thick periodic multilayer is replaced by a layer of the isotropic noncentrosymmetric material of width $w_{d}$; in other words, the finitely thick periodic multilayer is replaced by a homogeneous slab of the isotropic noncentrosymmetric medium of width $N\left(w_{p}+w_{d}\right)$. Let

$$
P_{\text {cross }}^{\prime}=\frac{1}{2 \eta_{0}}\left[\left|R_{(-)}^{\prime}-R_{(+)}^{\prime}\right|^{2}+\left|T_{(-)}^{\prime}-T_{(+)}^{\prime}\right|^{2}\right] \sin ^{2} \xi \cos ^{2} \xi
$$


be defined analogously to $P_{\text {cross }}$. We devised the quantity

$$
m_{\mathrm{rel}}=\frac{P_{\text {cross }}}{P_{\text {cross }}^{\prime}}=\frac{\left|R_{(-)}-R_{(+)}\right|^{2}+\left|T_{(-)}-T_{(+)}\right|^{2}}{\left|R_{(-)}^{\prime}-R_{(+)}^{\prime}\right|^{2}+\left|T_{(-)}^{\prime}-T_{(+)}^{\prime}\right|^{2}}
$$

as a relative metric of the effective in-plane anisotropy of the finitely thick periodic multilayer, with $m_{\text {rel }}>1$ denoting an enhancement of the effective in-plane anisotropy relative to a homogeneous layer of the isotropic noncentrosymmetric material of the same thickness and $m_{\mathrm{rel}}<1$ denoting a degradation of the effective in-plane anisotropy. From Eqs. (17) and (19), we also obtain that $m_{\text {rel }}=m_{\mathrm{abs}} / m_{\mathrm{abs}}^{\prime}$, where $m_{\mathrm{abs}}^{\prime}=P_{\text {cross }}^{\prime} / P_{\text {inc }}$. Both metrics $m_{\text {abs }}$ and $m_{\text {rel }}$ are similar to the ones defined elsewhere $^{39}$ for in-plane anisotropy and non-reciprocity.

Of the two metrics, the more significant is $m_{\text {rel }}$ since our primary intention is to enhance the effective in-plane anisotropy of the isotropic noncentrosymmetric material $(\mathrm{CdTe})$ by periodically interleaving its layers with ENG layers, whereas $m_{\text {abs }}$ plays a complementary role. We do not care much about the value of the polarization angle $\xi$ since it influences in the same way both the numerator and the denominator on the right side of (19); indeed, $m_{\text {rel }}$ is independent of $\xi$.

In contrast, the metric $m_{\mathrm{abs}}$ is proportional to $\sin ^{2} \xi \cos ^{2} \xi=\sin ^{2}(2 \xi) / 4$, indicating no change of the effective in-plane anisotropy when $\xi=0^{\circ}$ or $\xi=90^{\circ}-$ which is not surprising because the reflected and transmitted field phasors are then completely co-polarized with respect to the incident plane wave. For this reason, we chose to present data on $m_{\mathrm{abs}}$ here only for $\xi=45^{\circ}$, which is the polarization angle leading to maximal depolarization on reflection and transmission.

\section{B. Enhancement of effective in-plane anisotropy}

With the aim of realizing large values of $m_{\text {rel }}$, we first computed this metric as a function of the thickness ratio $w_{p} / w_{d}$ and the relative permittivity scalar $\varepsilon_{d}$ of the plasmonic ENG material for three different operational frequencies $f \in$ $\{30,60,90\} \mathrm{THz}$. The thickness $h$ of the finite periodic multilayer was kept fixed at $2500 \mathrm{~nm}$ and the number $N$ of bilayers equal to 20. In the density plots of $m_{\text {rel }}$ presented in Fig. 2, this metric is maximal nearly along the diagonal for all three selected frequencies. This is reasonable for the following reason: as the thickness ratio $w_{p} / w_{d}$ increases, the layers of the plasmonic ENG material become thinner than the layers of the isotropic noncentrosymmetric material; therefore, $\varepsilon_{d}$ must be more negative to enhance the in-plane anisotropy of the equivalent permittivity dyadic of the multilayer by the same degree and thus preserve the value of $m_{\text {rel }}$.

Furthermore, $m_{\text {rel }}$ can be remarkably high in magnitude for all three selected frequencies. Indeed, numerous combinations of $w_{p} / w_{d}$ and $\varepsilon_{d}$ yield a tenfold enhancement of inplane anisotropy compared to a homogeneous CdTe layer of thickness $h=2500 \mathrm{~nm}$. Finally, at higher frequencies, the peak of $m_{\text {rel }}$ with respect to $w_{p} / w_{d}$ for a fixed $\varepsilon_{d}$ is sharper but not necessarily higher.

Next, for each value of $N \in[1,20]$, we performed a greedy maximization ${ }^{40}$ of $m_{\text {rel }}$ with respect to both $w_{p}$ $\in[20,300] \mathrm{nm}$ and $w_{d} \in[100,200] \mathrm{nm}$ while keeping $\varepsilon_{d}$ $=-15$ fixed. Obviously, the overall thickness $h$ was left variable. Figure 3 provides the optimal pairs $\left(w_{p}, w_{d}\right)$ for three different operational frequencies $f \in\{30,60,90\}$ THz. At each of these three frequencies, the optimal values of $w_{p}$ and $w_{d}$ have similar convergence trends as $N$ increases. This

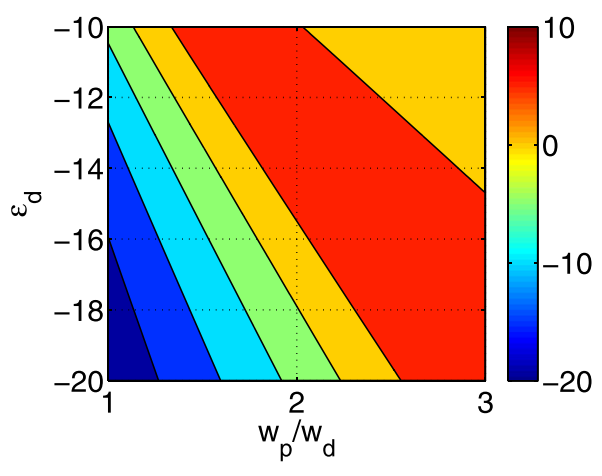

(a)

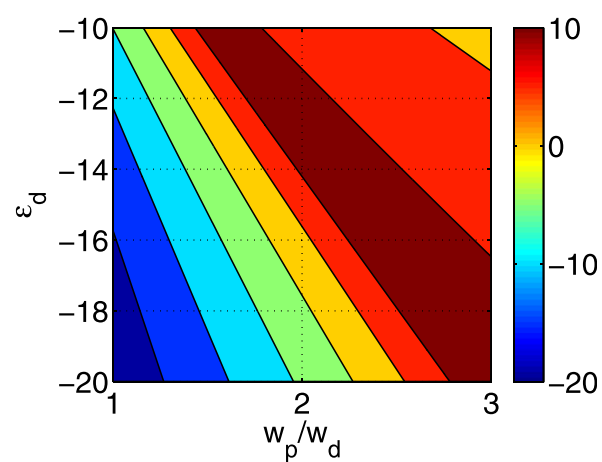

(b)
FIG. 2. Density plots of $10 \log \left(m_{\text {rel }}\right)$ as a function of $w_{p} / w_{d}$ and $\varepsilon_{d}$ for (a) $f=30 \mathrm{THz}$, (b) $f=60 \mathrm{THz}$, and (c) $f=90 \mathrm{THz}$, when $N=20, h=2500 \mathrm{~nm}$, and $E_{\mathrm{dc}}=1 \mathrm{MV} \mathrm{m}^{-1}$.

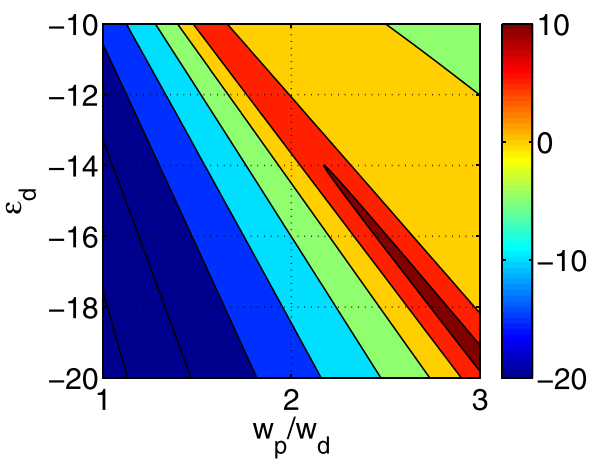

(c) 


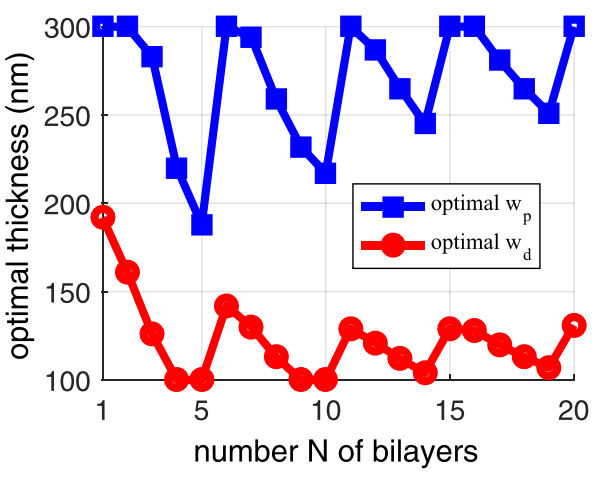

(a)

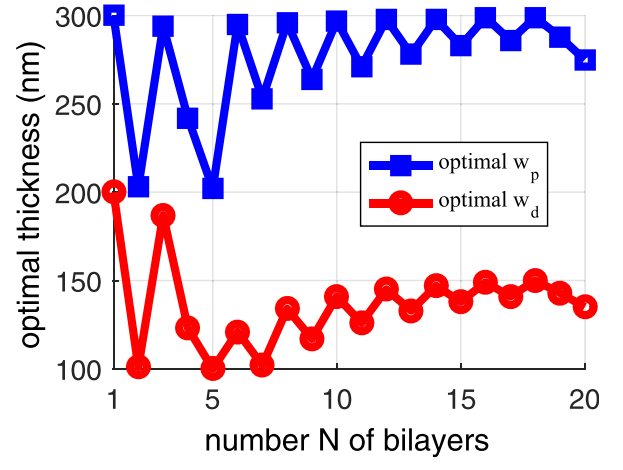

(b)

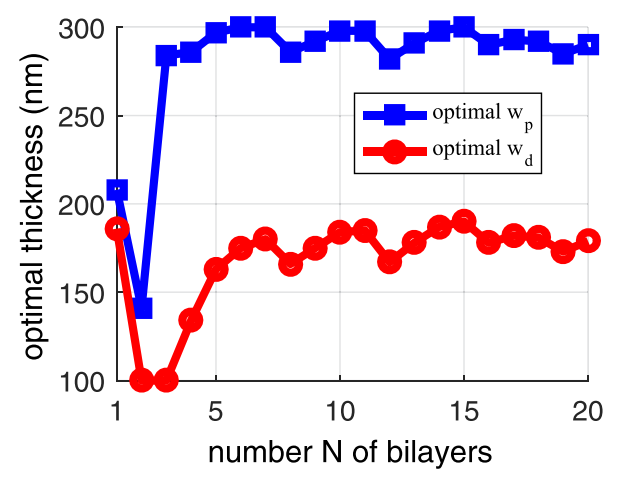

FIG. 3. Optimal values of $w_{p} \in$ $[20,300] \mathrm{nm}$ and $w_{d} \in[100,200] \mathrm{nm}$ needed to maximize $m_{\mathrm{rel}}$, as functions of $N$ for (a) $f=30 \mathrm{THz}$, (b) $f=60 \mathrm{THz}$, and (c) $f=90 \mathrm{THz}$, when $\varepsilon_{d}=-15$ and $E_{\mathrm{dc}}=1 \mathrm{MV} \mathrm{m}^{-1}$.

(c)

characteristic, combined with the fact that the $m_{\mathrm{rel}}$-maximization algorithm did not get stuck at the boundaries of the pre-set $w_{p}$ - and $w_{d}$-ranges, indicates that the enhancement of the effective in-plane anisotropy for the optimal configurations would be quite high. When it comes to the effect of the frequency, the data for optimal $w_{p}$ and $w_{d}$ exhibit more rapid oscillations with respect to $N$ for smaller $f$-because convergence cannot be achieved if $h$ is electrically small.

Figure 4 provides the high values of $m_{\text {rel }}$ after each greedy maximization leading to the optimal pairs $\left(w_{p}, w_{d}\right)$ presented in Fig. 3. Regardless of the operational frequency, $m_{\text {rel }}$ has a generally increasing trend with higher values of $N$.

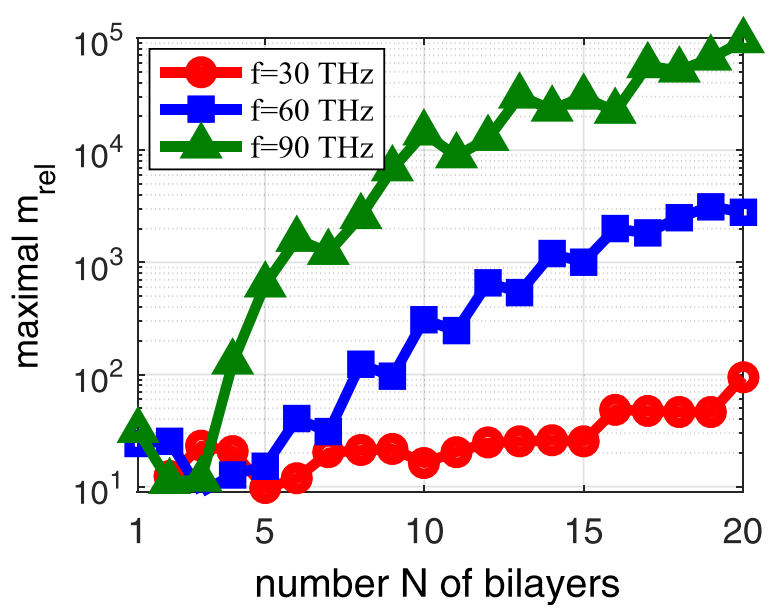

FIG. 4. Metric $m_{\text {rel }}$ as a function of $N$ for the optimal pairs $\left(w_{p}, w_{d}\right)$ presented in Fig. 3, when $f \in\{30,60,90\} \mathrm{THz}, \varepsilon_{d}=-15$, and $E_{\mathrm{dc}}=1 \mathrm{MV} \mathrm{m}^{-1}$.
Furthermore, $m_{\text {rel }}$ grows significantly with $f$ for $N>5$. The metric $m_{\mathrm{rel}}$ increases by one order of magnitude for $f=30 \mathrm{THz}$, but by four orders of magnitude for $f=90 \mathrm{THz}$, as $N$ increases from 2 to 20 , indicating thereby that the two types of layers interact much more strongly as they become electrically thicker. Thus, giant effective in-plane anisotropy is theoretically possible.

For each of the three selected operational frequencies in Fig. 4 , we determined the triad $\left(w_{p}, w_{d}, N\right)$ for the maximum value of $m_{\text {rel }}$. These optimal triads are

(I) $w_{p}=300 \mathrm{~nm}, w_{d}=131 \mathrm{~nm}$, and $N=20$, when $f=30$ $\mathrm{THz}$;

(II) $w_{p}=288 \mathrm{~nm}, w_{d}=143 \mathrm{~nm}$, and $N=19$, when $f=60$ $\mathrm{THz}$; and

(III) $w_{p}=290 \mathrm{~nm}, w_{d}=179 \mathrm{~nm}$, and $N=20$, when $f=90$ $\mathrm{THz}$.

The computed values of $m_{\mathrm{abs}}$ are plotted in Fig. 5 as functions of $E_{\mathrm{dc}}$ for the optimal triads I-III, when $\varepsilon_{d}=-15$ and $E_{\mathrm{dc}}=1 \mathrm{MV} \mathrm{m}^{-1}$ are fixed. Also, shown are values of $m_{\mathrm{abs}}$ when

(A) the entire finitely thick periodic multilayer is replaced by a homogeneous CdTe layer of thickness $h$;

(B) every plasmonic ENG layer in the finitely thick periodic multilayer is replaced by a complementary epsilon-positive (EPS) layer, i.e., when $\varepsilon_{d}=15$; and

(C) every plasmonic ENG material in the finitely thick periodic multilayer is replaced by a layer of air, i.e., when $\varepsilon_{d}=1$. 


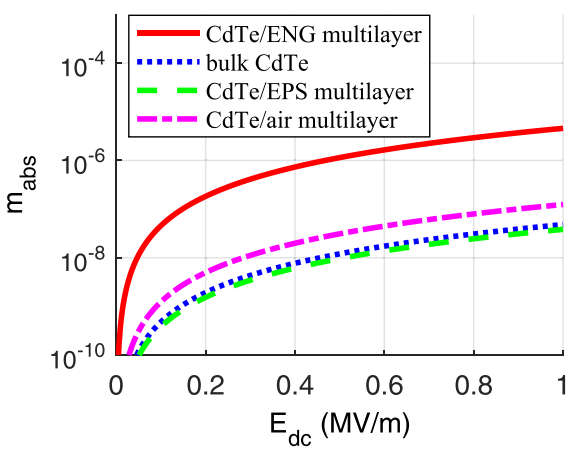

(a)

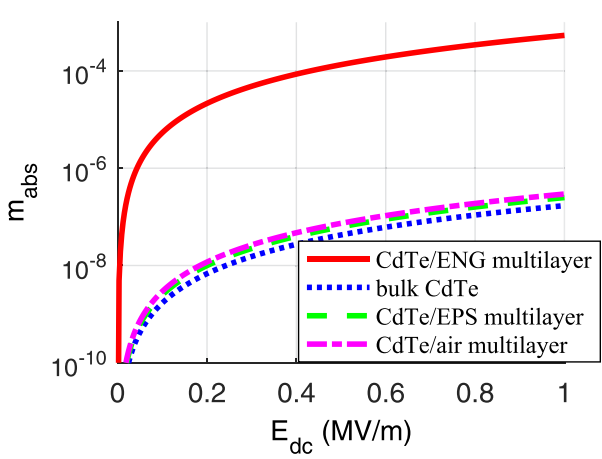

(b)

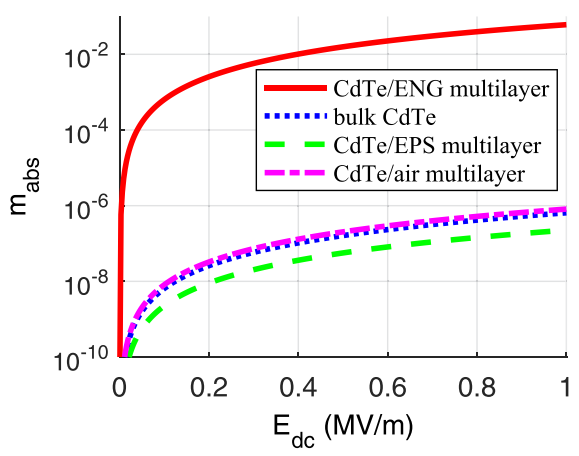

FIG. 5. Red solid curves: Metric $m_{\mathrm{abs}}$ as a function of $E_{\mathrm{dc}}$ when $\varepsilon_{d}=-15$, for the optimal triads $\left(w_{p}, w_{d}, N\right)$ gleaned from Fig. 4 to maximize $m_{\text {rel }}$ at (a) $f$ $=30 \mathrm{THz}\left(w_{p}=300 \mathrm{~nm}, w_{d}=131 \mathrm{~nm}\right.$, and $N=20)$, (b) $f=60 \mathrm{THz} \quad\left(w_{p}\right.$ $=288 \mathrm{~nm}, w_{d}=143 \mathrm{~nm}$, and $\left.N=19\right)$, and (c) $f=90 \mathrm{THz}\left(w_{p}=290 \mathrm{~nm}, w_{d}\right.$ $=179 \mathrm{~nm}$, and $N=20$ ). Data for the counterpart structures of types A (blue dotted curves), B (green dashed curves), and $\mathrm{C}$ (magenta dash-dotted curves) are also plotted.

(c)

Figure 5 shows that the effective in-plane anisotropies of the periodic CdTe/ENG, CdTe/CdTe, CdTe/EPS, and $\mathrm{CdTe} /$ air multilayers are enhanced on increasing $E_{\mathrm{dc}}$. The improved in-plane anisotropy of any of the four structures can be attributed to the direct proportionality of $\alpha$ to $E_{\mathrm{dc}}$, when $\left|r_{\mathrm{pc}} E_{\mathrm{dc}} n_{\mathrm{pc}}^{2}\right| \ll 1$. More importantly, Fig. 5 allows us to conclude that the in-plane anisotropy of the optimal CdTe/ ENG multilayer is superior to those of not only the homogeneous layer of CdTe (structure A) but also of the CdTe/EPS and CdTE/air multilayers (structures B and C, respectively) of the same overall thickness $h$. Clearly then, interleaving CdTe layers with layers of a material with $\varepsilon_{d}<0$ is crucial to realize giant effective in-plane anisotropy. The data in Figs. 4 and 5 support the contention that the spread between the effective in-plane anisotropies of the periodic CdTe/ENG multilayer on the one hand and the CdTe, CdTe/EPS, and $\mathrm{CdTe} / \mathrm{air}$ counterparts on the other hand widens as the frequency increases.

The most interesting feature of Fig. 5 is the evidence that maximization of $m_{\mathrm{rel}}$ also raises $m_{\mathrm{abs}}$ by a significant factor. In particular, for the highest frequency in consideration (i.e., $f=90 \mathrm{THz}$ ), we have $m_{\mathrm{abs}} \cong 10^{-1}$ for the periodic CdTe/ENG multilayer in Fig. 5(c), which means that the signal ratios $\left|\mathbf{E}_{\text {cross }}^{\text {ref }}\right| /\left|\mathbf{E}^{\text {inc }}\right|$ and $\left|\mathbf{E}_{\text {cross }}^{\text {trans }}\right| /\left|\mathbf{E}^{\text {inc }}\right|$ can be as high as $0.3<\sqrt{10^{-1}}$. At the lowest operational frequency considered (i.e., $f=30 \mathrm{THz}$ ), the value of $m_{\mathrm{abs}}$ of the periodic CdTe/ENG multilayer is smaller-but still considerably larger than that of the homogeneous CdTe layer of the same thickness.

Finally, we calculated $m_{\mathrm{abs}}$ of the finitely thick periodic $\mathrm{CdTe} / \mathrm{ENG}$ multilayers with the optimal triad $\left(w_{p}, w_{d}, N\right)$ labeled as I-III, as a function of $f \in[20,100] \mathrm{THz}$, when $\varepsilon_{d}=-15$ and $E_{\mathrm{dc}}=1 \mathrm{MV} \mathrm{m}^{-1}$. For comparison, we also evaluated $m_{\mathrm{abs}}$ for the counterpart structures of types A-C. For the first optimal triad (Fig. 6(a)) that maximizes $m_{\text {rel }}$ at $f=30 \mathrm{THz}$, a local maximum of $m_{\text {abs }}$ exists at $f=30 \mathrm{THz}$. In addition, and despite the oscillations, $m_{\mathrm{abs}}$ of the CdTe/ ENG multilayer remains larger than those of the counterpart structures A-C for almost all $f \in[20,100]$ THz. The second optimal triad (Fig. 6(b)) that maximizes $m_{\text {rel }}$ at $f=60 \mathrm{THz}$ has values of $m_{\mathrm{abs}}$ either comparable to or worse than those of the counterpart structures $\mathrm{A}-\mathrm{C}$ for $f<55 \mathrm{THz}$; however, the values of $m_{\mathrm{abs}}$ are significantly superior to those of the counterpart structures A-C for $f>55 \mathrm{THz}$. Finally, the third optimal triad (Fig. 6(c)) that maximizes $m_{\text {rel }}$ at $f=90 \mathrm{THz}$ has values of $m_{\text {abs }}$ either comparable to or worse than those of the counterpart structures $\mathrm{A}-\mathrm{C}$ for $f<85 \mathrm{THz}$; however, the values of $m_{\mathrm{abs}}$ are much higher than those of the counterpart structures A-C for $f>85 \mathrm{THz}$.

\section{Effect of dissipation and dispersion}

For the data presented in Sec. III B, we assumed that every ENG layer has a relative permittivity $\varepsilon_{d}$, which is purely real, negative, and independent of the frequency $f$. However, these assumptions are not realistic since plasmonic behavior is always accompanied by dissipation (or loss) and frequency dispersion. Therefore, we now propose actual materials that exhibit the required characteristics in the vicinity of the considered frequencies, and we quantify the effect of non-zero $\operatorname{Im}\left[\varepsilon_{d}\right]$ on the performance of the optimal periodic multilayers. 


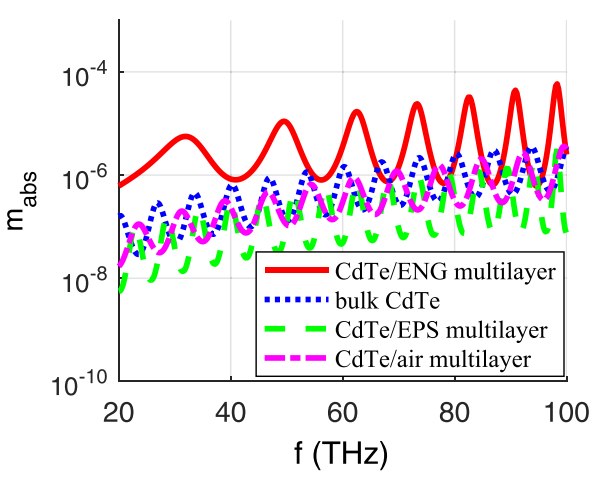

(a)

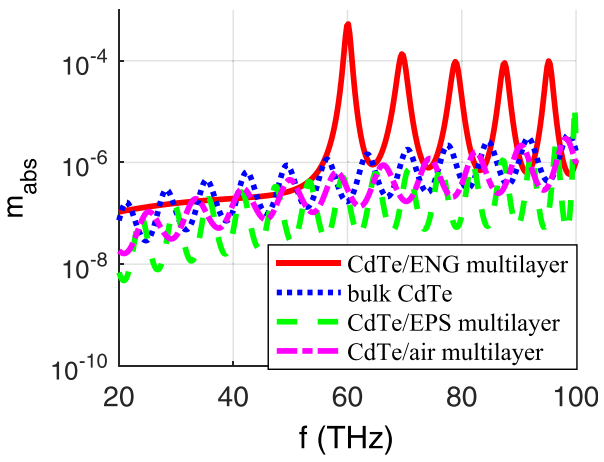

(b)

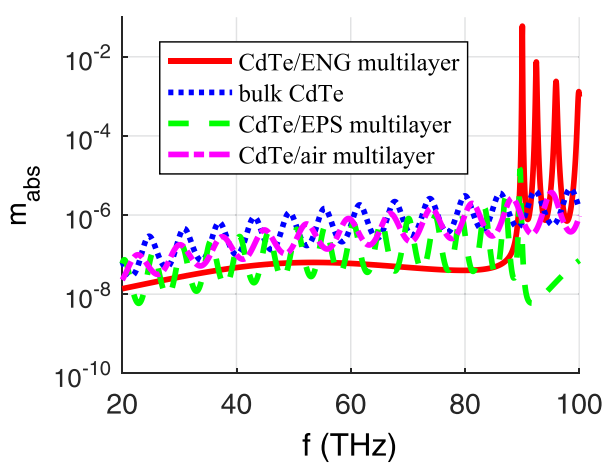

(c)

From the literature, ${ }^{41,42}$ it is clear that $\operatorname{Re}\left[\varepsilon_{d}\right]<0$ can be realized in the MWIR regime by suitably doping semiconductors. As an example, the relative permittivity of heavily doped indium arsenide (InAs) obeys the Drude model ${ }^{43,44}$

$$
\varepsilon_{\text {InAs }}(f)=\varepsilon_{\infty, I n A s}\left(1-\frac{f_{P, I n A s}^{2}}{f\left(f-i \gamma_{I n A s}\right)}\right), \quad f \in[20,30] \mathrm{THz}
$$

where $\varepsilon_{\infty, \text { InAs }}=11.9,{ }^{45} f_{P . \text { InAs }}=35.3 \mathrm{THz}$ [Ref. 43, Table 1, Film Sample 009], and $\gamma_{\text {InAs }}=1.44 \mathrm{THz}$ [Ref. 43, Table 1]. As another example, the relative permittivity of doped indium phosphide (InP) is the sum of a Drude term and a Lorentz term in the MWIR regime. ${ }^{46}$ Thus,

$$
\begin{aligned}
\varepsilon_{\operatorname{In} P}(f)= & \varepsilon_{\infty, \operatorname{In} P}\left(1-\frac{f_{P, \operatorname{In} P}^{2}}{f\left(f-i \gamma_{\operatorname{In} P}\right)}\right) \\
& +S_{\operatorname{In} P}\left(\frac{f_{F, \operatorname{In} P}^{2}}{f_{F, \operatorname{In} P}^{2}+i f \Gamma_{\operatorname{In} P}-f^{2}}\right), \quad f \in[20,30] \mathrm{THz},
\end{aligned}
$$

where $\varepsilon_{\infty, I n P}=9.55, f_{P, I n P}=39.3 \mathrm{THz}, \quad \gamma_{I n P}=2.76 \mathrm{THz}$, $S_{I n P}=3.83, f_{F, I n P}=9.13 \mathrm{THz}$, and $\Gamma_{I n P}=0.0057 \mathrm{THz}[\mathrm{Ref}$. 46, Table 2, Epilayer Sample 1]. In the same spectral regime, the relative permittivity of $4 \mathrm{H}$-silicon carbide ( $\mathrm{SiC}$ ) obeys the Lorentz model ${ }^{47,48}$

$$
\begin{aligned}
& \varepsilon_{S i C}(f)=\varepsilon_{\infty, S i C}\left(1+\frac{f_{L O, S i C}^{2}-f_{T O, S i C}^{2}}{f_{T O, S i C}^{2}+i f \Gamma_{S i C}-f^{2}}\right), \\
& f \in[20,30] \mathrm{THz},
\end{aligned}
$$

where $\varepsilon_{\infty, S i C}=6.5, f_{L O, S i C}=29.1 \mathrm{THz}, f_{T O, S i C}=23.9 \mathrm{THz}$, and $\Gamma_{\text {SiC }}=0.084 \mathrm{THz}$ [Ref. 47, Table 1, Sample 4].

As all data reported in Figs. 3-6 are premised on the assumption $\varepsilon_{d}=-15$, now we seek to find the frequencies under which the aforementioned three materials display this value of relative permittivity. The same or similar materials can be used for another value of $\varepsilon_{d}$ but at different operational frequencies. Figure 7 contains plots of the relative permittivities of (20)-(22) as functions of the frequency $f$. The vertical dashed lines in the figure mark the frequencies $f_{\text {InAs }} \cong 23.43 \mathrm{THz}, f_{\text {InP }} \cong 24.65 \mathrm{THz}$, and $f_{S i C} \cong 25.59 \mathrm{THz}$ at which the condition $\operatorname{Re}\left[\varepsilon_{d}\right]=-15$ is fulfilled. In Fig. 7(a), the variations of $\operatorname{Re}\left[\varepsilon_{I n A s}(f)\right]$ and $\operatorname{Re}\left[\varepsilon_{I n P}(f)\right]$ with frequency are similar and slowly increasing with $f$ in the neighborhoods of $f_{\text {InAs }}$ and $f_{\text {InP }}$, respectively, but $\operatorname{Re}\left[\varepsilon_{S i C}(f)\right]$ drops rapidly as $f$ reduces from $f_{S i C}$. However, satisfaction of the condition $\operatorname{Re}\left[\varepsilon_{d}\right]=-15$ is accompanied by $\operatorname{Im}\left[\varepsilon_{d}\right]<0$ in Fig. 7(b). The least accompanying value of $\operatorname{Im}\left[\varepsilon_{d}\right]<0$ is that of $\mathrm{SiC}$, and the most is that of InP. We notice that we employ the proposed materials at low MWIR frequencies (20 $\mathrm{THz}<f<30 \mathrm{THz}$ ); however, this does not constitute a significant limitation. Indeed, for alternative desirable $\operatorname{Re}\left[\varepsilon_{d}\right]$, the dielectric characteristics of the same media can fulfill the ENG requirement at higher frequencies.

Values of the metric $m_{\text {rel }}$ are presented in Fig. 8 when the ENG material is either heavily doped InAs, doped InP, or 4H$\mathrm{SiC}$, and the frequency lies in the vicinity of $f_{\text {InAs }}, f_{\text {InP }}$, and $f_{S i C}$. For each of these three frequencies, optimal values of $w_{p}$, $w_{d}$, and $N$ were determined (in the same way that Fig. 2 is obtained) as follows: (a) $w_{p}=297 \mathrm{~nm}, w_{d}=129 \mathrm{~nm}$, and $N=9$ for $f=f_{\text {InAs }}$ and $\varepsilon_{d}=-15$ (approximated by heavily doped InAs); (b) $w_{p}=282 \mathrm{~nm}, w_{d}=122 \mathrm{~nm}$, and $N=9$ for 


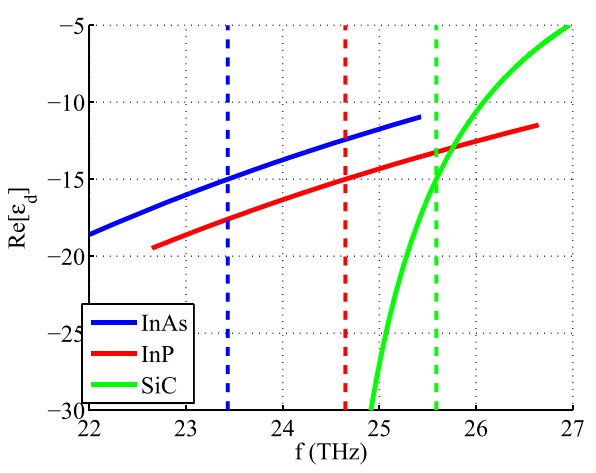

(a)

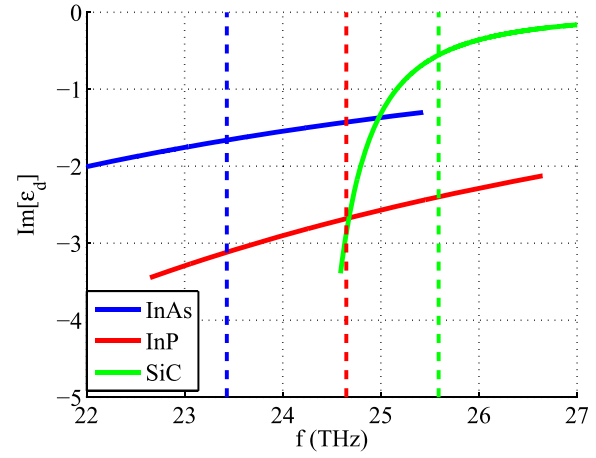

(b)
FIG. 7. (a) Real and (b) imaginary parts of the relative permittivity of doped InAs, InP, and $\mathrm{SiC}$ as functions of the frequency $f$. The vertical dashed lines indicate the frequencies at which $\operatorname{Re}\left[\varepsilon_{d}\right]=-15$. $f=f_{\text {InP }}$ and $\varepsilon_{d}=-15$ (approximated by doped InP); and (c) $w_{p}=300 \mathrm{~nm}, w_{d}=132 \mathrm{~nm}$, and $N=8$ for $f=f_{\text {SiC }}$ and $\varepsilon_{d}=-15$ (approximated by $4 \mathrm{H}-\mathrm{SiC}$ ). Furthermore, data are also provided in Fig. 8 for (i) the ENG material being made artificially non-dissipative (i.e., $\operatorname{Im}\left[\varepsilon_{d}\right] \equiv 0 \forall f$ ) and (ii) the ENG material being ideal (i.e., $\varepsilon_{d} \equiv-15 \forall f$ ).

We clearly observe in Fig. 8(a) that dissipation reduces $m_{\text {rel }}$ by a factor of about 4 when the ENG material is heavily doped InAs in comparison to the ideal case. The value of $m_{\text {rel }}$ for the artificially non-dissipative case coincides with its value for the ideal case only for $f=f_{\text {InAs }}$ but is less otherwise. In the case of doped InP, the situation is expected to be worse due to the higher dissipation indicated in Fig. 7(b). Indeed, the relative enhancement of the anisotropy in Fig. 8(b) is only one eighth of that achieved for the ideal case. When $4 \mathrm{H}-\mathrm{SiC}$ is incorporated as the ENG material, Fig. 8(c) indicates a 12-fold effective anisotropy enhancement over CdTe alone, which is more than half the enhancement in the ideal case. In other words, the use of $4 \mathrm{H}-\mathrm{SiC}$ as the ENG material can substantially enhance the anisotropy in the response of the periodic $4 \mathrm{H}-\mathrm{SiC} / \mathrm{CdTe}$ multilayer over that of the homogeneous CdTe layer of the same total thickness at the lower MWIR frequencies.

Heavily doped InAs, doped InP, and $4 \mathrm{H}-\mathrm{SiC}$ are only a few representative examples of actual ENG materials with dissipative and dispersive plasmonic properties at midinfrared frequencies. Alternatively, one can use stacks of doped graphene, ${ }^{17,49}$ which combines negative real effective permittivity with low dissipation. Another approach would be the neutralization of the inevitable dissipation by using both active $e^{50,51}$ and dissipative layers. If the thicknesses of the two layers are equal $\left(=w_{d} / 2\right)$ and the relative permittivities are conjugates of each other, then an efficient design comprised of Parity-Time symmetric bilayers ${ }^{11,52}$ alternating with CdTe layers could be proposed.

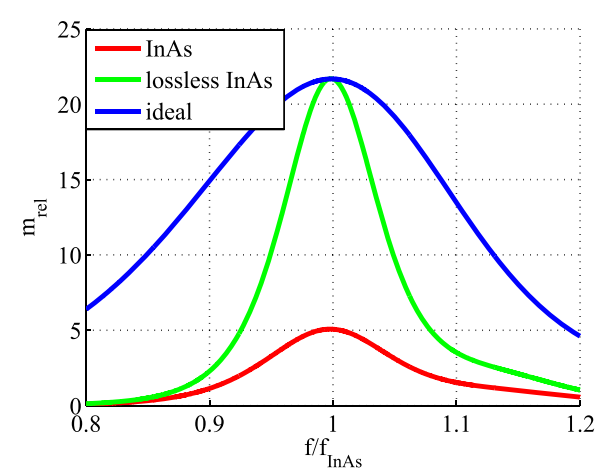

(a)

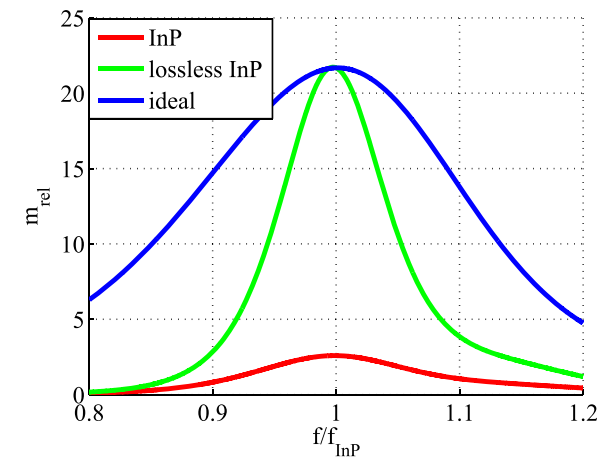

(b)

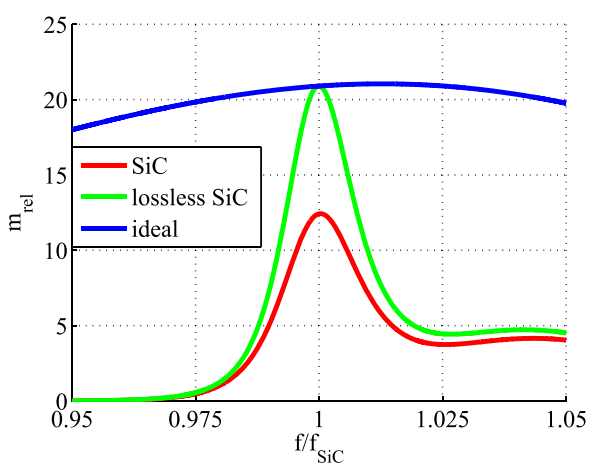

(c)
FIG. 8. The relative metrics $m_{\text {rel }}$ of several finitely thick periodic multilayers (actual, nondissipative dispersive, and ideal) as functions of the relative operational frequency for the three considered materials: (a) heavily doped InAs with $w_{p}=297 \mathrm{~nm}, w_{d}=129 \mathrm{~nm}$, and $N=9$; (b) doped InP with $w_{p}=282 \mathrm{~nm}$, $w_{d}=122 \mathrm{~nm}$, and $N=9$; and (c) $4 \mathrm{H}-$ $\mathrm{SiC}$ with $w_{p}=300 \mathrm{~nm}, w_{d}=132 \mathrm{~nm}$, and $N=8$. 


\section{CONCLUDING REMARKS}

When an isotropic noncentrosymmetric material is subjected to a uniform dc electric field aligned along a certain fixed direction, its dielectric characteristics in the midwavelength and long-wavelength infrared regimes exhibit anisotropy in the plane to which the dc electric field is normal. This electro-optic effect can be controlled by the magnitude of an external dc electric field. However, the in-plane anisotropy is quite weak because the magnitude of the dc electric field must be kept below its breakdown limit and the electro-optic coefficient is small. To remedy such a weakness, we proposed to use finitely thick periodic multilayers comprising electrically thin layers of a biased noncentrosymmetric material (more specifically, CdTe) alternating with electrically thin layers of an isotropic plasmonic epsilonnegative material. The negative real part of the relative permittivity of the latter material will diminish the diagonal terms of the relative permittivity dyadic of the biased noncentrosymmetric material while leaving untouched the offdiagonal ones.

Our conjecture has been verified via numerical simulations of the reflection and transmission of a linearly polarized plane wave that is normally incident on the finitely thick periodic multilayer, the dc electric field being oriented parallel to the direction of propagation of the incident plane wave. We determined that the cross-polarized power density of the reflected and the transmitted plane waves can be much larger for the periodic CdTe/ENG multilayer than for a homogeneous CdTe layer of the same thickness. This enhancement of the effective in-plane anisotropy can be maximized by optimal selections of the layer thicknesses and the number of unit cells. Under the assumption of the ENG material being non-dissipative, we can report a stable enhancement of the effective in-plane anisotropy by an average of three orders of magnitude, which is fully controllable by external dc bias. The presence of dissipation in real ENG materials will depress the degree of enhancement, but it will still be significant. We believe that our results may inspire further experimental and theoretical efforts in the field of $\mathrm{THz}$ plasmonics combined with signal processing applications, such as modulation, buffering, polarization transformation, and switching.

${ }^{1}$ R. W. Boyd, Nonlinear Optics, 3rd ed. (Elsevier, Burlington, MA, 2008). ${ }^{2}$ I. P. Kaminow, "Measurements of the electrooptic effect in CdS, ZnTe, and GaAs at 10.6 microns," IEEE J. Quantum Electron. 4, 23-26 (1968).

${ }^{3}$ J. E. Kiefer and A. Yariv, "Electro-optic characteristics of CdTe at 3.39 and 10.6 $\mu$," Appl. Phys. Lett. 15, 26-27 (1969).

${ }^{4}$ R. O. Bell, "Review of optical applications of CdTe," Rev. de Phys. Appl. 12, 391-399 (1977).

${ }^{5}$ T. A. Maldonado, "Electro-optic modulators," in Handbook of Optics II, 2nd ed., edited by M. Bass, E. W. Van Stryland, D. R. Williams, and W. L. Wolfe (McGraw-Hill, New York, NY, 1995), Chap. 13.

${ }^{6}$ C. A. Valagiannopoulos, N. L. Tsitsas, A. Lakhtakia, and A. Burger, "Pockels cover for switchable control of the reflection from a grounded, isotropic, lossy dielectric slab," J. Appl. Phys. 117, 083105 (2015).

${ }^{7}$ C. J. Johnson, G. L. Herrit, and E. R. Mueller, "Electro-optic modulator applications," in CdTe and Related Compounds; Physics, Defects, Heteroand Nano-structures, Crystal Growth, Surfaces and Applications, edited by R. Triboulet and P. Siffert (Elsevier, Oxford, United Kingdom, 2010), Chap. 11D.

${ }^{8}$ A. K. Ghatak and K. Thyagarajan, Optical Electronics (Cambridge University Press, Cambridge, United Kingdom, 1989)
${ }^{9}$ S. Hrabar, "Waveguide experiments to characterize properties of SNG and DNG metamaterials," in Metamaterials Physics and Engineering Explorations, edited by N. Engheta and R. W. Ziolkowksi (Wiley, Hoboken, NJ, USA, 2006), Chap. 3.

${ }^{10}$ D. Schurig, J. J. Mock, and D. R. Smith, "Electric-field-coupled resonators for negative permittivity metamaterials," Appl. Phys. Lett. 88, 041109 (2006).

${ }^{11}$ C. A. Valagiannopoulos, "Optical PT-symmetric counterparts of ordinary metals," IEEE J. Sel. Top. Quantum Electron. 22(5), 89-97 (2016).

${ }^{12} \mathrm{~A}$. Herpin, "Calcul du pouvoir réflecteur d'un système stratifié quelconque," Comptes Rendue Acad. Sci. (Paris) 225, 182-183 (1947).

${ }^{13}$ S. M. Rytov, "Electromagnetic properties of a finely stratified medium," Sov. Phys. JETP 2, 466-475 (1956)

${ }^{14}$ A. Lakhtakia, "Constraints on effective constitutive parameters of certain bianisotropic laminated composite materials," Electromagnetics 29, 508-514 (2009).

${ }^{15}$ A. A. Orlov, S. V. Zhukovsky, I. V. Iorsh, and P. A. Belov, "Controlling light with plasmonic multilayers," Photonics Nanostruct.: Fundam. Appl. 12, 213-230 (2014).

${ }^{16}$ I. V. Iorsh, I. S. Mukhin, I. V. Shadrivov, P. A. Belov, and Y. S. Kivshar, "Hyperbolic metamaterials based on multilayer graphene structures," Phys. Rev. B 87, 075416 (2013).

${ }^{17}$ I. S. Nefedov, C. A. Valagiannopoulos, and L. A. Melnikov, "Perfect absorption in graphene multilayers," J. Opt. (Bristol) 15, 114003 (2013).

${ }^{18}$ S. Vuković, J. J. Miret, C. J. Zapata-Rodríguez, and Z. Jaksić, "Oblique surface waves at an interface between a metal-dielectric superlattice and an isotropic dielectric," Phys. Scri. 149, 014041 (2012).

${ }^{19}$ C. A. Valagiannopoulos and N. L. Tsitsas, "Integral equation analysis of a low-profile receiving planar microstrip antenna with a cloaking superstrate," Radio Sci. 47, RS2022 (2012).

${ }^{20}$ J. B. Pendry, A. J. Holden, D. J. Robbins, and W. J. Stewart, "Low frequency plasmons in thin-wire structures," J. Phys.: Condens. Matter 10, 4785-4809 (1998).

${ }^{21}$ L. Solymar and E. Shamonina, Waves in Metamaterials (Oxford University Press, Oxford, United Kingdom, 2009)

${ }^{22}$ F. Xu, Y. Bai, L. Qiao, H. Zhao, and J. Zhou, "Realization of negative permittivity of $\mathrm{Co}_{2} \mathrm{Z}$ hexagonal ferrite and left-handed property of ferrite composite material," J. Phys. D: Appl. Phys. 42, 025403 (2009).

${ }^{23}$ K. L. Gordon, J. H. Kang, C. Park, P. T. Lillehei, and J. S. Harrison, "A novel negative dielectric constant material based on phosphoric acid doped poly(benzimidazole)," J. Appl. Polymer Sci. 125, 2977-2985 (2012).

${ }^{24} \mathrm{~L}$. Thylen, "Creating a high quality factor negative epsilon photonic material from two level resonant systems," Appl. Phys. A 119, 33-39 (2015).

${ }^{25}$ Nonlinear, Tunable and Active Metamaterials, edited by I. V. Shadrivov, M. Lapine, and Y. S. Kivshar (Springer, Cham, Switzerland, 2015).

${ }^{26}$ R. C. Jones, "New calculus for the treatment of optical systems. VIII. Electromagnetic theory," J. Opt. Soc. Am. 46, 126-131 (1956).

${ }^{27}$ I. Abdulhalim, " $2 \times 2$ Matrix summation method for multiple reflections and transmissions in a biaxial slab between two anisotropic media," Opt. Commun. 163, 9-14 (1999).

${ }^{28}$ J. A. Reyes and A. Lakhtakia, "Electrically controlled optical bandgap in a structurally chiral material," Opt. Commun. 259, 164-173 (2006).

${ }^{29} \mathrm{H}$. C. Chen, Theory of Electromagnetic Waves: A Coordinate-Free Approach (McGraw-Hill, New York, NY, 1983)

${ }^{30}$ J. Lajzerowicz, L. Verger, F. Mathy, and M. Cuzin, "Power switching with a new material: CdTe:Cl,” Proc. SPIE 1632, 217-222 (1992).

${ }^{31}$ H.-Y. Kim, J. O. Sofo, D. Velegol, M. W. Cole, and G. Mukhopadhyay, "Static polarizabilities of dielectric nanoclusters," Phys. Rev. A 72, 053201 (2005).

${ }^{32}$ J. Faist, F. Capasso, D. L. Sivco, C. Sirtori, A. L. Hutchinson, and A. Y. Cho, "Quantum cascade laser," Science 264, 553-556 (1994).

${ }^{33}$ S. M. George, "Atomic layer deposition: An overview," Chem. Rev. 110, 111-131 (2010).

${ }^{34}$ X. Li, G. Zhang, X. Bai, X. Sun, X. Wang, E. Wang, and H. Dai, "highly conducting graphene sheets and Langmuir-Blodgett films," Nat. Nanotechnol. 3, 538-542 (2008).

${ }^{35}$ C. J. Zapata-Rodriguez, D. Pastor, J. J. Miret, and S. Vukovic, "Uniaxial epsilon-near-zero metamaterials: From superlensing to double refraction," J. Nanophotonics 8, 083895 (2014).

${ }^{36}$ J. Lekner, "Reflection by absorbing periodically stratified media," J. Opt. (Bristol) 16, 035104 (2014).

${ }^{37}$ A. Lakhtakia, "Reflection from a semi-infinite rugate filter," J. Mod. Opt. 58, 562-565 (2011). 
${ }^{38}$ M. W. McCall and A. Lakhtakia, "Explicit expressions for spectral remittances of axially excited chiral sculptured thin films," J. Mod. Opt. 51, 111-127 (2004).

${ }^{39} \mathrm{C}$. A. Valagiannopoulos, "How nonreciprocal is an effective permittivity matrix?," Microwave Opt. Technol. Lett. 56, 2018-2021 (2014).

${ }^{40}$ C. A. Valagiannopoulos, N. L. Tsitsas, and A. H. Sihvola, "Hiding a bump on a PEC plane by using an isotropic lossless dielectric layer," IEEE Trans. Antennas Propag. 62, 5706-5714 (2014).

${ }^{41}$ R. Stanley, "Plasmonics in the mid-infrared," Nat. Photonics 6, 409-411 (2012).

${ }^{42}$ A. Boltasseva and H. A. Atwater, "Low-loss plasmonic metamaterials," Science 331, 290-291 (2011).

${ }^{43}$ S. Law, D. C. Adams, A. M. Taylor, and D. Wasserman, "Mid-infrared designer metals," Opt. Express 20, 12155-12165 (2012).

${ }^{44}$ Y. Zhong, S. D. Malagari, T. Hamilton, and D. Wasserman, "Review of mid-infrared plasmonic materials," J. Nanophotonics 9, 093791 (2015).

${ }^{45}$ See http://refractiveindex.info/ for the permittivity of InAs; accessed January 9, 2017.

${ }^{46}$ M. E. A. Panah, O. Takayama, S. V. Morozov, K. E. Kudryavtsev, E. S. Semenova, and A. V. Lavrinenko, "Highly doped InP as a low loss plasmonic material for mid-IR region,” Opt. Express 24, 29077-29088 (2016).

${ }^{47}$ T. E. Tiwald, J. A. Woollam, S. Zollner, J. Christiansen, R. B. Gregory, T. Wetteroth, S. R. Wilson, and A. R. Powell, "Carrier concentration and lattice absorption in bulk and epitaxial silicon carbide determined using infrared ellipsometry," Phys. Rev. B 60, 11464-11474 (1999).

${ }^{48}$ J. D. Caldwell, L. Lindsay, V. Giannini, I. Vurgaftman, T. L. Reinecke, S. A. Maier, and O. J. Glembocki, "Low-loss, infrared and terahertz nanophotonics using surface phonon polaritons," Nanophotonics 4, 44-68 (2015).

${ }^{49}$ M. Mattheakis, C. A. Valagiannopoulos, and E. Kaxiras, "Epsilon-nearzero behavior from plasmonic Dirac point: Theory and realization using two-dimensional materials," Phys. Rev. B 94, 201404 (2016).

${ }^{50}$ D. Rodrigo, O. Limaj, D. Janner, D. Etezadi, F. J. Garcia de Abajo, V. Pruneri, and H. Altug, "Mid-infrared plasmonic biosensing with graphene," Science 349, 165-168 (2015).

${ }^{51}$ D. Li and C. Z. Ning, "All-semiconductor active plasmonic system in midinfrared wavelengths," Opt. Express 19, 14594-14603 (2011).

${ }^{52} \mathrm{C}$. A. Valagiannopoulos, F. Monticone, and A. Alù, "PT-symmetric planar devices for field transformation and imaging," J. Opt. (Bristol) 18, 044028 (2016). 